\title{
Variational Methods in Semilinear Wave Equation with Nonlinear Boundary Conditions and Stability Questions
}

\author{
A. Nowakowski ${ }^{1}$
}

Received: 2 February 2016 / Revised: 1 April 2016 / Published online: 20 April 2016

(c) The Author(s) 2016. This article is published with open access at Springerlink.com

\begin{abstract}
We present variational approach to the semilinear equation of the vibrating string $x_{t t}(t, y)-\Delta x(t, y)+l(t, y, x(t, y))=0$ in bounded domain and certain type of nonlinearity on the boundary. To this effect we derive new dual variational method. Next the question of stability of solutions with respect to initial conditions is discussed.
\end{abstract}

Keywords Dual variational method - Semi-linear wave equation · Dissipation and sources term on the boundary

\section{Introduction}

We assume $\Omega$ to be an open bounded domain in $\mathbf{R}^{n}, n \geq 2$, with sufficiently smooth boundary $\Gamma$. In the paper we study, over a finite interval $[0, T], T<\infty$ ( $T$-arbitrary but fixed $)$ the second order semilinear wave equation with nonlinearity containing source terms in the interior of the domain and dissipation and source terms on its boundary:

$$
\begin{aligned}
& x_{t t}(t, y)-\Delta x(t, y)+l(t, y, x(t, y))=0, \text { in }(0, T) \times \Omega, \\
& \partial_{\nu} x(t, y)+x(t, y)+g\left(t, y, x_{t}(t, y)\right)=h(t, y, x(t, y)) \text { on } \Sigma=(0, T) \times \Gamma, \\
& x(0, \cdot)=x^{0}(\cdot) \in H^{1}(\Omega), x_{t}(0, \cdot)=x^{1}(\cdot) \in L_{2}(\Omega) .
\end{aligned}
$$

Studying the above problems have long history see e.g. [2-4,6,8-10]. In most of these papers nonlinearities do not depend on $(t, y)$ and are of special type and they concern well-posedness of the system given by (1) on the finite energy space, i.e. $H^{1}(\Omega) \times L_{2}(\Omega)$. The well-posedness included existence and uniqueness of both local and global solutions. However, as it is shown in [9] if we want to admit more general nonlinearities then we cannot expect well-posedness in Hadamard sense. The main difficulty and the novelty of the problems considered here relate to the presence of the boundary nonlinear term $h(t, y, x)$. This difficulty has to do

A. Nowakowski annowako@math.uni.lodz.pl

1 Faculty of Math \& Computer Sciences, University of Lodz, Banacha 22, 90-238 Lodz, Poland 
with the fact that Lopatinski condition does not hold for the Neumann problem. The above translates into the fact that in the absence of the damping, the linear map $h \rightarrow\left(x(t), x_{t}(t)\right)$, is not bounded from $L^{2}(\Sigma) \rightarrow H^{1}(\Omega) \times L_{2}(\Omega)$, unless the dimension of $\Omega$ is equal to one. More details on this problem are discussed in [4,9].

In this paper we study a global existence question over a finite interval $[0, T], T<\infty$ for different types of nonlinearities $l, g, h$, than in $[2,4,9,20]$ and depending on $(t, y)$ and so we are not interested in well-posedness in Hadamard sense. But, we will prove stability of the system with respect to initial conditions i.e. continuous dependence (in some new sense) with respect to initial conditions.

It is well known that in general the nonlinearities $l, h$ may drive the solution of (1) to blow up in finite time [2,12,14,23,25-28] (see also discussion in [9] in context of appearance of the dissipation $g$ ). In order to exclude such a case we impose some interaction between structure of $l$, quantity of initial condition $x^{0}(\cdot) \in H^{1}(\Omega)$ and length of time $T$ (see the assumption As). The importance of the problem with nonlinearity on the boundary appears in optimal control theory see e.g. $[9,10,15-17]$ and the references therein. The problems like (1) were studied mostly by topological method, semigroup theory or monotone operator theory (see $[3,9,10]$ for discussion about that). As it is known to the author there are no papers studying (1) (with nonlinearity on the boundary) by variational method except the author's papers [19-22] where a different type of nonlinearities were investigated (linear combination of monotone function or difference of two functions with strong assumptions). We would like to stress that just using the variational approach the dissipation term $g$ on the boundary has no influence on the existence of solution to (1). This is why we do not discuss the interaction between dissipation and source on the boundary as it is usual done while approaching other methods (see e.g. [2,4,6,9,24]). What is essential in the method used here is that, we consider first the equation

$$
\partial_{v} x(t, y)+x(t, y)+g\left(t, y, x_{t}(t, y)\right)=h(t, y, x(t, y)) \text { on } \Sigma=(0, T) \times \Gamma
$$

and put $H=\frac{1}{2} x^{2}$, which is a convex function. Convexity is exploited in the paper in different forms and just convexity eliminates other assumptions. The general nonlinearity of the interior source has the form:

$$
l(t, y, x)= \pm F_{x}(t, y, x)-G_{x}(t, y, x), \quad x \in \mathbf{R}, \quad(t, y) \in(0, T) \times \Omega
$$

where $F, G$ are $C^{1}$ and $F$ is additionally a convex functions with respect to third variable. It turns out that in this case a size of initial conditions is not essential, however some restrictions on initial conditions are hidden in $(\mathbf{H} \Gamma)$ and (As) below.

We shall study (1) by variational method. However, we shall not consider (1) as the EulerLagrange equations of any functional. Instead, we shall investigate two functionals one in interior domain

$$
\begin{aligned}
J^{F}(x)= & \int_{0}^{T} \int_{\Omega}\left(\frac{1}{2}|\nabla x(t, y)|^{2}-\frac{1}{2}\left|x_{t}(t, y)\right|^{2}+F_{x}(t, y, x(t, y))\right. \\
& \left.-x(t, y) G_{x}(t, y, x(t, y))\right) d y d t-\left(x^{0}(\cdot), x^{1}(\cdot)\right)_{L_{2}(\Omega)},
\end{aligned}
$$

and the second on the boundary

$$
\begin{aligned}
J_{\Gamma}(x)= & \int_{0}^{T} \int_{\Gamma}(H(t, y, x(t, y)) \\
& +H^{*}\left(t, y,-\partial_{\nu} x(t, y)-g\left(t, y, x_{t}(t, y)\right)+h(t, y, x(t, y))\right) \\
& \left.-x(t, y)\left(-\partial_{\nu} x(t, y)-g\left(t, y, x_{t}(t, y)\right)+h(t, y, x(t, y))\right)\right) d y d t
\end{aligned}
$$


defined on some subspaces of the space $C\left([0, T] ; H^{1}(\Omega)\right)$ and $C\left([0, T] ; H^{1}(\Gamma)\right)$, respectively, discussed below. $H^{*}$ is the Fenchel conjugate to $H$. Just studying functionals (3) and (4) we do not need to use energy functionals for system (1), which are standard tools to study relations between nonlinearity, length of time $T$ and quantity of initial condition $x^{0}$.

Our purpose is to investigate (1) by studying critical points of functional (3). To this effect we apply a new duality approach. As it is easy to see, functional (3) is unbounded in $C\left([0, T] ; H^{1}(\Omega)\right)$ and this is a reason for which we are looking for critical points of $J^{F}$ different than extremum or minmax type. Our aim is to find a nonlinear subspace $X^{F}$ of $C\left([0, T] ; H^{1}(\Omega)\right)$ and study (3) just only on $X^{F}$. In last section we prove stability of solutions of (1) when $x^{0}(\cdot)$ and $x^{1}(\cdot)$ are changing in a suitable way.

First we will study the equation

$$
\begin{aligned}
& \partial_{\nu} x(t, y)+x(t, y)+g\left(t, y, x_{t}(t, y)\right)=h(t, y, x(t, y)) \text { on }(0, T) \times \Gamma, \\
& x(0, \cdot)=x^{0}(\cdot) \in H^{1}(\Gamma),
\end{aligned}
$$

with the help of the functional $J_{\Gamma}(x)$ (see (4)) - Sect. 3. Next we solve

$$
\begin{aligned}
& x_{t t}(t, y)-\Delta x(t, y)+l(t, y, x(t, y))=0 \text { in }(0, T) \times \Omega, \\
& x(t, y)=x_{\Gamma}(t, y) \text { on }(0, T) \times \Gamma, x_{\Gamma}(0, \cdot)=x^{0}(\cdot) \text { on } \Gamma, \\
& x(0, \cdot)=x^{0}(\cdot) \in H^{1}(\Omega), x_{t}(0, \cdot)=x^{1}(\cdot) \in L_{2}(\Omega),
\end{aligned}
$$

where $x_{\Gamma}$ is a solution of (5), with the help of the functional $J^{F}(x)$ (see (3))-Sect. 4 for the case $l(t, y, x)=F_{x}(t, y, x)-G_{x}(t, y, x)$.

\section{Main Results}

We will focus on the case when $n \geq 3$ ( $n=2$ being the least interesting, since the concept of criticality of Sobolev's embedding is much less pronounced). First we formulate results concerning problem (5), its relation (see [1]) to the functional $J_{\Gamma}(x)$ and problem (6). To this effect we need to explain what we mean by $x^{0}(\cdot) \in H^{1}(\Gamma)$ in (5), $x^{0}(\cdot) \in H^{1}(\Omega)$ in (6) and the normal derivative $\partial_{\nu} x(t, y)$ in (5) and in (4). To this effect define $\widetilde{H}^{1}(\Gamma)=\left\{x \in H^{1}(\Omega):\left.x\right|_{\Gamma} \in H^{1}(\Gamma)\right\}$ and we shall consider the space $C\left([0, T] ; \widetilde{H}^{1}(\Gamma)\right)$. Therefore by the normal derivative $\partial_{v} x(t, y)$ in (5) and also in (4) we mean normal derivative of a function $x(\cdot, \cdot) \in C\left([0, T] ; H^{1}(\Omega)\right) \cap C\left([0, T] ; \widetilde{H}^{1}(\Gamma)\right)$. Thus let $x^{0} \in \widetilde{H}^{1}(\Gamma)$. We use a set

$$
U^{t}=\left\{x: x(t, \cdot)=x^{0}(\cdot)+t w(\cdot), \quad w \in \widetilde{H}^{1}(\Gamma)\right\}, \quad t \in[0, T]
$$

and the functional

$$
\begin{aligned}
J_{\Gamma}^{t}(x)= & \int_{\Gamma}\left(H(t, y, x(t, y))+H^{*}\left(t, y,-\partial_{\nu} x(t, y)-g\left(t, y, x_{t}(t, y)\right)\right.\right. \\
& \left.+h(t, y, x(t, y)))-x(t, y)\left(-\partial_{\nu} x(t, y)-g\left(t, y, x_{t}(t, y)\right)+h(t, y, x(t, y))\right)\right) d y
\end{aligned}
$$

considered on $U^{t}, t \in[0, T]$ and $H(t, y, x)=\frac{1}{2} x^{2}$. That means we are looking for solutions to (5) in $U^{t}, t \in[0, T]$. Therefore, if such a solution belonging to $U^{t}, t \in[0, T]$, exists it is a strong solution to (5).

Assumptions concerning problem (5): 
$(\mathbf{H} \Gamma) h(\cdot, \cdot, v)$ is measurable in $[0, T] \times \Gamma$ for each $v \in \mathbf{R}, h(t, y, \cdot)$ is continuous in $\mathbf{R}$, for $(t, y) \in[0, T] \times \Gamma$ and satisfies the growth condition

$$
-h(t, y, x) \geq c(t, y)|x|^{\alpha}+d(t, y), t \in[0, T],(t, y, x) \in(0, T) \times \Gamma \times \mathbf{R},
$$

where $\alpha>1$ any fixed, $c(t, y)>0, d(t, y) \geq 0,(t, y) \in(0, T) \times \Gamma, h(\cdot, \cdot, x(\cdot, \cdot)) \in L_{2}(\Sigma)$ for $x(t, \cdot) \in U^{t}, t \in[0, T] . g(\cdot, \cdot, s)$ is measurable in $(0, T) \times \Gamma, s \in \mathbf{R}, g(t, y, \cdot)$ is continuous in $\mathbf{R}, g(\cdot, \cdot, x(\cdot, \cdot)) \in L_{2}(\Sigma)$ for $x(t, \cdot) \in U^{t}, t \in[0, T]$ and satisfies the growth condition

$$
g(t, y, s) \geq a(t, y)|s|^{r}+b(t, y),(t, y, s) \in(0, T) \times \Gamma \times \mathbf{R}
$$

for some $a, b \in L^{\infty}((0, T) \times \Gamma), a \geq 0, b \geq 0, r \geq 1$.

Remark 1 The unique restriction on nonlinearity is that only the growth on $g$ and $h$ are imposed. Moreover we would like to stress that $x^{0} \in \widetilde{H}^{1}(\Gamma)$, thus we impose a little bit more regularity on initial condition $x^{0} \in H^{1}(\Omega)$.

Being inspired by Brezis-Ekeland [7] (see also [18]) we formulate the variational principle for problem (5).

Proposition $1 x_{\Gamma}(t, \cdot) \in U^{t}, t \in[0, T]$ is a solution to (5) if and only if $x_{\Gamma}(t, \cdot)$ affords a minimum to the functional $J_{\Gamma}^{t}$ defined on $U^{t}, t \in[0, T]$ and $J_{\Gamma}^{t}\left(x_{\Gamma}\right)=0$.

Proposition 2 Under assumption $(\boldsymbol{H} \Gamma)$ there is an $x_{\Gamma}(t, \cdot) \in U^{t}$ which affords a minimum to the functional $J_{\Gamma}^{t}$ defined on $U^{t}, t \in[0, T]$ and $J_{\Gamma}^{t}\left(x_{\Gamma}\right)=0$.

Corollary 1 The problem (5) has a solution $x_{\Gamma}(t, \cdot) \in U^{t}, t \in[0, T]$.

Remark 2 It is worth to note that the damping term $g$ may be zero i.e. it has not influence on existence of solutions to (5) in $U^{t}, t \in[0, T]$. Usually (see [4,5,9]), damping term $g$ is monotonic and source term $h$ is smooth but not necessarily monotonic, in our case we have, the source term of the form $x-h$ and dissipation term $g$ is only continuous and satisfying some growth condition. The reason is that we use quite different approach to solve (1). We would like to stress, once more, that just convexity of $x \rightarrow H(t, y, x)=\frac{1}{2} x^{2}$ is very important here and plays a crucial role in the proof - it eliminates a role of damping term $g$. Just different method used here allows significantly relaxed assumptions concerning problem (1). We assume only that the term $h$ is only continuous in $x$ and satisfies the growth condition (8). Moreover we admit (this is done first time - to the best knowledge of the author) dependence of $h$ on $(t, y)$ (compare [5,20] and literature therein). In our case solutions of (5) are independent of solutions of (6). It is necessary to stress that solution to (5) exists on each finite interval $[0, T]$, as long as assumption $(\mathbf{H} \Gamma)$ is satisfied on this interval, i.e. our assumptions depend on the interval $[0, T]$.

We introduce the definition of a weak solution to (6):

Definition 1 (weak solution) By a weak solution to (6), defined on a given interval $[0, T]$, we mean a function $x \in U$, where

$$
\begin{aligned}
U=\left\{x: x \in C\left([0, T] ; H^{1}(\Omega)\right), \quad \frac{\partial x}{\partial t} \in C\left([0, T] ; L_{2}(\Omega)\right), x(0, y)=x^{0}(y),\right. \\
\left.x_{t}(0, y)=x^{1}(y), \quad y \in \Omega, x(t, y)=x_{\Gamma}(t, y),(t, y) \in \Sigma\right\}
\end{aligned}
$$


such that for all $\varphi \in U_{\varphi}$

$$
\begin{aligned}
& \int_{0}^{T} \int_{\Omega}\left(-x_{t} \varphi_{t}+\nabla x \nabla \varphi\right) d y d t+\int_{0} \int_{\Omega} l \varphi d y d t \\
& =\int_{0}^{T} \int_{\Gamma}(-x-g+h) \varphi d y d t \\
& \quad-\int_{\Omega} x_{t}(T, y) \varphi(T, y) d y+\int_{\Omega} x_{t}(0, y) \varphi(0, y) d y,
\end{aligned}
$$

where

$$
U_{\varphi}=\left\{\varphi: \varphi \in C\left([0, T] ; H^{1}(\Omega)\right), \quad \frac{\partial \varphi}{\partial t} \in C\left([0, T] ; L_{2}(\Omega)\right),\left.\varphi\right|_{\Sigma} \in L_{2}(\Sigma)\right\} .
$$

We shall consider $U$ with a topology induced by the norm

$$
\|x\|_{U}=\|x\|_{C\left([0, T] ; H^{1}(\Omega)\right)}+\left\|x_{t}\right\|_{C\left([0, T] ; L_{2}(\Omega)\right)} .
$$

Remark 3 The weak solution considered in this paper is stronger than e.g. in [5] where $x \in$ $C_{w}\left([0, T] ; H^{1}(\Omega)\right), \frac{\partial x}{\partial t} \in C\left([0, T] ; L_{2}(\Omega)\right)\left(C_{w}([0, T] ; Y)\right.$ denotes the space of weakly continuous functions with values in a Banach space $Y$ ).

The main contribution of this paper is a relaxation of assumptions on nonlinearities $l$ and $h$. Moreover, in spite of lack of uniqueness results we have global (for given finite interval $[0, T]$ ) existence theorem to (1) and continuous dependence of solutions with respect to initial data in the following sense:

Definition 2 For given sequences $\left\{x_{n}^{0}\right\} \subset \widetilde{H}^{1}(\Gamma),\left\{x_{n}^{1}\right\} \subset L_{2}(\Omega)$, converging to $\bar{x}^{0}$ in $\widetilde{H}^{1}(\Gamma), \bar{x}^{1}$ in $L_{2}(\Omega)$, respectively, there is a subsequence of $\left\{x_{n}\right\}$-solutions to (1) corresponding to $\left\{x_{n}^{0}\right\},\left\{x_{n}^{1}\right\}$, which we denote again by $\left\{x_{n}\right\}$ weakly convergent in $H^{1}((0, T) \times \Omega)$ and strongly in $L_{2}((0, T) \times \Omega)$ to an element $\bar{x} \in U$ being a solution to (1) corresponding to $\bar{x}^{0}, \bar{x}^{1}$.

To formulate assumptions concerning equation (6) we need to recall some theorems from [13] - linear case of (6) which we use as a starting point to study (6).

Theorem 1 Let $\bar{G}(\cdot, \cdot) \in L^{1}\left(0, T ; L_{2}(\Omega)\right), x_{\Gamma}(t, \cdot) \in U^{t}, t \in[0, T]$, is a solution to (5), $x^{0} \in \widetilde{H}^{1}(\Gamma), x^{1} \in L_{2}(\Omega)$ with the compatibility condition: $x_{\Gamma}(0, y)=x^{0}(y), y \in \Gamma$. Then there exists $\bar{x}$ being a unique weak solution to

$$
\begin{aligned}
& x_{t t}(t, y)-\Delta x(t, y)=\bar{G}(t, y), \\
& x(0, y)=x^{0}(y), x_{t}(0, y)=x^{1}(y), y \in \Omega, \\
& x(t, y)=x_{\Gamma}(t, y),(t, y) \in \Sigma
\end{aligned}
$$

and such that $\bar{x} \in C\left([0, T] ; H^{1}(\Omega)\right), \bar{x}_{t} \in C\left([0, T] ; L_{2}(\Omega)\right), \partial_{\nu} \bar{x} \in L_{2}(\Sigma)$,

$$
\begin{aligned}
& \|\bar{x}\|_{C\left([0, T] ; H^{1}(\Omega)\right)} \leq C\left(\|\bar{G}\|_{L^{1}\left(0, T ; L_{2}(\Omega)\right)}+\left\|x_{\Gamma}\right\|_{H^{1}(\Sigma)}+\left\|x^{0}\right\|_{H^{1}(\Omega)}+\left\|x^{1}\right\|_{L_{2}(\Omega)}\right), \\
& \left\|\bar{x}_{t}\right\|_{C\left([0, T] ; L_{2}(\Omega)\right)} \leq D\left(\|\bar{G}\|_{L^{1}\left(0, T ; L_{2}(\Omega)\right)}+\left\|x_{\Gamma}\right\|_{H^{1}(\Sigma)}+\left\|x^{0}\right\|_{H^{1}(\Omega)}+\left\|x^{1}\right\|_{L_{2}(\Omega)}\right)
\end{aligned}
$$

with some $C>0, D>0$ independent of a choice of $\bar{G}(\cdot, \cdot) \in L^{1}\left(0, T ; L_{2}(\Omega)\right)$. 
Shortly the solution $\bar{x}$ from the above theorem may be estimated by

$$
\begin{aligned}
& \|\bar{x}\|_{C\left([0, T] ; H^{1}(\Omega)\right)} \leq C\|\bar{G}\|_{L^{1}\left(0, T ; L_{2}(\Omega)\right)}+E^{w}, \\
& \left\|\bar{x}_{t}\right\|_{C\left([0, T] ; L_{2}(\Omega)\right)} \leq D\|\bar{G}\|_{L^{1}\left(0, T ; L_{2}(\Omega)\right)}+A^{w},
\end{aligned}
$$

where $E^{w}=C\left(\left\|x_{\Gamma}\right\|_{H^{1}(\Sigma)}+\left\|x^{0}\right\|_{H^{1}(\Omega)}+\left\|x^{1}\right\|_{L_{2}(\Omega)}\right)$ and $A^{w}=D\left(\left\|x_{\Gamma}\right\|_{H^{1}(\Sigma)}+\right.$ $\left.\left\|x^{0}\right\|_{H^{1}(\Omega)}+\left\|x^{1}\right\|_{L_{2}(\Omega)}\right)$ and in the paper we will just use the last estimations.

Everywhere below constants $C$ and $D$ will always denote those occurring in (10), (11). Note that then $\|\bar{x}\|_{U} \leq(C+D)\|\bar{G}\|_{L^{1}\left(0, T ; L_{2}(\Omega)\right)}+\left(E^{w}+A^{w}\right)$

Assumptions concerning Eq. (1).

(As) Let $F$ and $G$ be functions of the variable $(t, y, x) \in[0, T] \times \Omega \times \mathbf{R}$. Assume that

(i) for any $x \in \mathbf{R}, F(\cdot, \cdot, x)$ and $G(\cdot, \cdot, x)$ are measurable,

(ii) for any $(t, y), F(t, y, \cdot), G(t, y, \cdot)$ are continuously differentiable,

(iii) for any $(t, y), F(t, y, \cdot)$ is convex,

(iv) $F(t, y, x) \geq a(t, y) x+b(t, y),(t, y, x) \in[0, T] \times \Omega \times \mathbf{R}$, for some $a, b \in$ $L^{1}\left(0, T ; L_{2}(\Omega)\right), a>0$,

(v) assume that our original nonlinearity $l$ (see (6)) has the form

$$
l=F_{x}-G_{x}
$$

or

$$
l=-F_{x}-G_{x}
$$

and that there exist constants $E_{F}, E_{G}$ such that

$$
\left\|F_{x}(x)\right\|_{L^{1}\left(0, T ; L_{2}(\Omega)\right)} \leq E_{F},\left\|G_{x}(x)\right\|_{L^{1}\left(0, T ; L_{2}(\Omega)\right)} \leq E_{G}, \quad x \in X^{F}
$$

(we use the notation $K_{x}(z)=K_{x}(t, y, z(t, y))$ ), where

$$
X^{F}=\left\{v \in U:\|v\|_{U} \leq(C+D)\left(E_{F}+E_{G}\right)+\left(E^{w}+A^{w}\right)\right\} .
$$

For every $x \in X^{F}$ consider a weak solution $\tilde{x} \in U$ of the problem

$$
\begin{aligned}
& \tilde{x}_{t t}(t, y)-\Delta \widetilde{x}(t, y)=-l(t, y, x(t, y)) \text { in }(0, T) \times \Omega, \\
& \widetilde{x}(t, y)=x_{\Gamma}(t, y) \text { on }(0, T) \times \Gamma, x_{\Gamma}(0, \cdot)=x^{0}(\cdot) \text { on } \Gamma, \\
& \tilde{x}(0, \cdot)=x^{0}(\cdot) \in \widetilde{H}^{1}(\Gamma), \widetilde{x}_{t}(0, \cdot)=x^{1}(\cdot) \in L_{2}(\Omega) .
\end{aligned}
$$

Define the map $\mathcal{H}$ assigning to $x \in X^{F}$ a weak solution $\tilde{x} \in U$ of the above linear problem. Let us put $\bar{X}^{F}=\mathcal{H}\left(X^{F}\right)$.

Theorem 2 (Main theorem). Under $(\boldsymbol{A s})$ and $(\boldsymbol{H} \Gamma)$ there exists $\bar{x} \in X^{F}$ such that

$$
J^{F}(\bar{x})=\sup _{x \in X^{F}} J^{F}(x)
$$

and $\bar{x}$ is a weak solution to (6).

Remark 4 We would like to stress that the result obtained in the paper is of global type-for each given, but fixed $T<\infty$. However assumption (As) shows us that there is a certain relation between the type of nonlinearity and the quantity of $T$, i.e. $\left\|F_{x}(x)\right\|_{L^{1}\left(0, T ; L_{2}(\Omega)\right)}$, $\left\|G_{x}(x)\right\|_{L^{1}\left(0, T ; L_{2}(\Omega)\right)}$ have to be bounded in some ball of $U \cap L^{1}(0, T ; L q(\Omega)), q>1$. Moreover, we do not admit the case $T=\infty$. The known results concerning existence of 
global solutions to (1) (without uniqueness and damping term) assume that nonlinearity $l$ is locally Lipschitz and sublinear at infinity or of polynomial growth $|s|^{p-1} s$ ( $p$ such that $\left.H^{1}(\Omega) \subset L_{p+1}(\Omega)\right)$ with additional boundedness for initial data (see e.g. [9]). We do not assume that $l$ is locally Lipschitz from $H^{1}(\Omega) \rightarrow L_{p+1}(\Omega)$, as $x \rightarrow l(x)$ is only continuous in R. Instead, we assume the special structure of $l$ (see (12)) - a combination of monotone function and continuous ones. Moreover the assumptions: $F_{x}(x), G_{x}(x)$ are bounded in some ball imply growth conditions for $l$. Some type of boundedness for initial data are hidden in the definitions of the set $X^{F}$. We would like to stress that $l$ is only continuous in $x$, usually $l$ is at least of $C^{1}$ (see e.g. [4,5,9]). Moreover we are looking for solutions in the bounded set $X^{F}$, so all solutions are bounded in the norm of $C\left([0, T] ; H^{1}(\Omega)\right)$. This is a case which is often assumed to get global solution (see [4,9] and references therein).

We ilustrate the above theorem (its assumptions) by an example.

Example 1 Put $n=3$. Assume that $\Omega$ is a ball with center at zero and volume $1 / 2$ and let $T=1 / 2$. We can assume that our constants $C=1 / 4$ and $D=1 / 4$. Put $g=0$ and $h(t, y, x)=-t^{2} \cos \left(y_{1} y_{2} y_{3}\right)\left|x^{5}\right|$. Then assumptions $(\mathbf{H} \Gamma)$ are satisfied. Let $F(t, y, x)=$ $\sqrt{t} \sin \left(y_{1} y_{2} y_{3}\right) x^{4}$ and $G(t, y, x)=(1 / \sqrt[4]{t}) y_{2} \tan x, x^{0}=0, x^{1}=1 / 8$. Hence for $E^{w}$ we can take $1 / 4$ and similarly for $A^{w}=1 / 4$ and let us take $E_{F}=1 / 2, E_{G}=1 / 2$ so $X^{F}=\left\{v \in U:\|v\|_{U} \leq(1 / 2)(1 / 2+1 / 2)+(1 / 4+1 / 4)\right\}$. Thus for each $v \in X^{F}$

$$
\max _{(0,1 / 2) \times \Omega}|v(t, y)| \leq 1,
$$

therefore $\left\|F_{x}(x)\right\|_{L^{1}\left(0, T ; L_{2}(\Omega)\right)} \leq 1 / 2,\left\|G_{x}(x)\right\|_{L^{1}\left(0, T ; L_{2}(\Omega)\right)} \leq 1 / 2$, for $x \in X^{F}$. Hence we infer that the assumptions (As) are satisfied too.

We have no uniqueness of solutions to (1), but a kind of continuous dependence on initial data is still possible.

Theorem 3 Assume $(\boldsymbol{H} \Gamma)$ and $(\boldsymbol{A s})$. Let $\left\{x_{n}^{0}\right\},\left\{x_{n}^{1}\right\}$ be given sequences in $\widetilde{H}^{1}(\Gamma), L_{2}(\Omega)$ respectively, converging to $\bar{x}^{0}, \bar{x}^{1}$ in $H^{1}(\Omega), L_{2}(\Omega)$ respectively and such that

$$
\begin{aligned}
\left\|x_{n}^{0}\right\|_{C\left([0, T] ; H^{1}(\Omega)\right)} & \leq C\left(E_{F}+E_{G}\right)+E^{w}, \\
\left\|x_{n}^{1}\right\|_{C\left([0, T] ; L_{2}(\Omega)\right)} & \leq D\left(E_{F}+E_{G}\right)+A^{w}, n=1,2 \ldots
\end{aligned}
$$

Then there is a subsequence of $\left\{x_{n}\right\}$-solutions to (1) corresponding to $\left\{x_{n}^{0}\right\},\left\{x_{n}^{1}\right\}$, which we denote again by $\left\{x_{n}\right\}$ weakly convergent in $H^{1}((0, T) \times \Omega)$ and strongly in $L_{2}((0, T) \times \Omega)$ to an element $\bar{x} \in U$ being a solution to (1) corresponding to $\bar{x}^{0}, \bar{x}^{1}$.

Let $F^{*}$ be the Fenchel conjugate of $F$. Define a dual to $J^{F}$ functional

$$
J_{D}^{F}: H^{1}((0, T) \times \Omega) \times H^{1}((0, T) \times \Omega)^{n} \times L_{2}\left(\left(0, T, L_{2}(\Omega)\right) \rightarrow \mathbf{R},\right.
$$

as:

$$
\begin{aligned}
J_{D}^{F}(p, q, z)= & -\int_{0}^{T} \int_{\Omega} F^{*}\left(t, y,\left(-p_{t}(t, y)+\operatorname{div} q(t, y)+z(t, y)\right)\right) d y d t \\
& -\frac{1}{2} \int_{0}^{T} \int_{\Omega}|q(t, y)|^{2} d y d t+\frac{1}{2} \int_{0}^{T} \int_{\Omega}|p(t, y)|^{2} d y d t \\
& -(\bar{x}(T, \cdot), p(T, \cdot))_{L_{2}(\Omega)}-\int_{\Sigma} x_{\Gamma}(t, y)\langle q(t, y), v(y)\rangle d y d t,
\end{aligned}
$$


where $\langle\cdot, \cdot\rangle$ is the inner product in $\mathbf{R}^{n}$ and $v=\left(v_{1}, \ldots, v_{n}\right)$ is the unit outward normal to $\Gamma$ and $\bar{x}$ is the minimizer of $J$ (see Theorem 2). Now we can formulate theorem which gives us additional informations on solutions to (1) important in classical mechanics. This theorem is absolutely new for problem (1).

Theorem 4 (Variational principle and duality result) Assume (As). Let $\bar{x} \in X^{F}$ be such that $J^{F}(\bar{x})=\sup _{x \in X^{F}} J^{F}(x)$. Then there exists $(\bar{p}, \bar{q}) \in H^{1}((0, T) \times \Omega) \times H^{1}((0, T) \times \Omega)^{n}$ such that for a.e. $(t, y) \in(0, T) \times \Omega$,

$$
\begin{aligned}
& \bar{p}(t, y)=\bar{x}_{t}(t, y), \\
& \bar{q}(t, y)=\nabla \bar{x}(t, y), \\
& -\bar{p}_{t}(t, y)+\operatorname{div} \bar{q}(t, y)-l(t, y, \bar{x}(t, y))=0
\end{aligned}
$$

and

$$
J^{F}(\bar{x})=J_{D}^{F}(\bar{p}, \bar{q}, \bar{z}),
$$

where

$$
\bar{z}=G_{x}(t, y, \bar{x}(t, y)) .
$$

The proofs of theorems are given in Sects. 3 and 4. They consist of several steps. First we prove Propositions 1, 2 and Corollary 1, i.e. we solve problem (5). Next we prove Theorem 2 (Main theorem).

\section{Proof of Existence for Problem (5)}

As mentioned before, the main difficulty of the problem under study is the fact that the Neumann problem does not satisfy Lopatinski condition and therefore, the map from the boundary data in $L_{2}(\Sigma)$ into finite energy space is not bounded (unless dimension of $\Omega$ is equal to one). In order to cope with this problem in [4,9] a regularizing term—strongly monotone dissipation is introduced, whose effect is to 'force' the Lopatinski condition. We follow, in quite, different way. We convert the problem (5) into variational one and this allow us to omit the meaning of the dissipation term. However, the price we pay for that is the form of nonlinearity for the boundary source term equals $h-x$. We start with the proof of Proposition 1

Proof of Proposition 1 It is a simple consequence of the equivalence of the following two relations in $(0, T) \times \Gamma$ :

$$
\begin{aligned}
& \text { (i) }-\partial_{\nu} \bar{x}(t, y)-g\left(t, y, \bar{x}_{t}(t, y)\right)+h(t, y, \bar{x}(t, y)) \in \partial H(t, y, \bar{x}(t, y)), \\
& \text { (ii) } H(t, y, \bar{x}(t, y))+H^{*}\left(t, y,-\partial_{\nu} \bar{x}(t, y)-g\left(t, y, \bar{x}_{t}(t, y)\right)+h(t, y, \bar{x}(t, y))\right) \\
& =\bar{x}(t, y)\left(-\partial_{\nu} \bar{x}(t, y)-g\left(t, y, \bar{x}_{t}(t, y)\right)+h(t, y, \bar{x}(t, y))\right)
\end{aligned}
$$

and the inequality

$$
\begin{aligned}
& H(t, y, x(t, y))+H^{*}\left(t, y,-\partial_{\nu} x(t, y)-g\left(t, y, x_{t}(t, y)\right)+h(t, y, x(t, y))\right) \\
& \quad \geq x(t, y)\left(-\partial_{\nu} x(t, y)-g\left(t, y, x_{t}(t, y)\right)+h(t, y, x(t, y))\right)
\end{aligned}
$$

which holds for all $x(t, \cdot) \in U^{t}, t \in[0, T]$. 
Let us notice that the sets $U^{t}$ are nonempty. We will study the functional $J_{\Gamma}^{t}(x)$ on $U^{t}, t \in[0, T]$ and we prove under hypothesis $(\mathbf{H} \Gamma)$ that there exists a minimum to the functional $J_{\Gamma}^{t}$, i.e. the proof of Proposition 2.

Proof of Proposition 2 Let us notice that $J_{\Gamma}^{t}(x(t, \cdot))$ is bounded below in $U^{t}, t \in[0, T]$, in fact, $J_{\Gamma}^{t}(x(t, \cdot)) \geq 0$ and weakly lower semicontinuous in $U^{t}, t \in[0, T]$. Moreover, let us observe, by $(\mathbf{H} \Gamma), J_{\Gamma}^{t}(x(t, \cdot)) \rightarrow \infty$ when $\|x(t, \cdot)\|_{H^{1}(\Gamma)} \rightarrow \infty$. Really, it is enough to notice that for $t \in[0, T]$ and $x \in U^{t}$

$$
\begin{aligned}
J_{\Gamma}^{t}(x(t, \cdot))= & \frac{1}{2} \int_{\Gamma}\left(x(t, y)+\partial_{\nu} x(t, y)+g\left(t, y, x_{t}(t, y)\right)-h(t, y, x(t, y))\right)^{2} d y \\
\geq & \frac{1}{2} \int_{\Gamma}\left(x(t, y)+\partial_{\nu} x(t, y)+a(t, y)\left|x_{t}(t, y)\right|^{r}\right. \\
& \left.+b(t, y)+c(t, y)|x(t, y)|^{\alpha}+d(t, y)\right)^{2} d y .
\end{aligned}
$$

From (21) we infer that $\left\|x_{n}(t, \cdot)\right\|_{L_{2}(\Gamma)}$ and $\left\|x_{t n}(t, \cdot)\right\|_{L_{2}(\Gamma)}$ are bounded for minimizing sequence $\left\{x_{n}(t, \cdot)\right\}$ of $J_{\Gamma}^{t}$ and next from

$$
\begin{aligned}
J_{\Gamma}^{t}(x(t, \cdot)) \geq & \frac{1}{2}\left(\|\nabla x(t, \cdot) v\|_{L_{2}}^{2}\right. \\
& \left.-\left\|a(t, \cdot)\left|x_{t}(t, \cdot)\right|^{r}+b(t, \cdot)+c(t, \cdot)|x(t, \cdot)|^{\alpha}+d(t, \cdot)\right\|_{L_{2}}^{2}\right)
\end{aligned}
$$

it follows that $\left\|\nabla x_{n}(t, \cdot)\right\|_{L_{2}(\Gamma)}$ is bounded. Thus, there is a subsequence of $\left\{x_{n}(t, \cdot)\right\}$ (which we again denote by $\left.\left\{x_{n}(t, \cdot)\right\}\right)$ such that it is weakly convergent to some $x_{\Gamma}$ in $H^{1}(\Gamma)$ and pointwise convergent to it. Therefore, for each $t \in[0, T], \liminf _{n \rightarrow \infty} J_{\Gamma}^{t}\left(x_{n}(t, \cdot)\right) \geq$ $J_{\Gamma}^{t}\left(x_{\Gamma}(t, \cdot)\right)$. Now let us define the dual functional to $J_{\Gamma}^{t}, t \in[0, T]$, by

$$
\begin{aligned}
J_{\Gamma D}^{t}(x)= & \int_{\Gamma}\left(H(t, y,-x(t, y))+H^{*}\left(t, y, \partial_{\nu} x(t, y)-g\left(t, y,-x_{t}(t, y)\right)\right.\right. \\
& +h(t, y,-x(t, y)))+x(t, y)\left(\partial_{\nu} x(t, y)\right. \\
& \left.\left.-g\left(t, y,-x_{t}(t, y)\right)+h(t, y,-x(t, y))\right)\right) d y .
\end{aligned}
$$

It is clear that $J_{\Gamma}^{t}(x(t, \cdot))=J_{\Gamma D}^{t}(-x(t, \cdot))$ for all $x(t, \cdot) \in U^{t}$ and so

$$
\inf _{x \in U^{t}} J_{\Gamma}^{t}(x(t, \cdot))=\inf _{x \in U^{t}} J_{\Gamma D}^{t}(-x(t, \cdot)), t \in[0, T] .
$$

By the duality theory for convex functionals (see [11,18]) we have that inf ${ }_{x \in U^{t}} J_{\Gamma}^{t}(x(t, \cdot))=$ $-\inf _{x \in U^{t}} J_{\Gamma D}^{t}(x(t, \cdot)), t \in[0, T]$. Hence we infer that $J_{\Gamma}^{t}\left(x_{\Gamma}(t, \cdot)\right)=0$.

Proof of Corollary 1 This is a direct consequence of two above Propositions 1 and 2.

\section{Proof of Existence for Problem (6)}

Consider equation (with $x_{\Gamma} \in H^{1}(\Sigma)$ being a solution to (5))

$$
\begin{aligned}
& x_{t t}(t, y)-\Delta x(t, y)+F_{x}(t, y, x(t, y))-G_{x}(t, y, x(t, y))=0, \text { in }(0, T) \times \Omega, \\
& x(t, y)=x_{\Gamma}(t, y),(t, y) \in \Sigma, \\
& x(0, \cdot)=x^{0}(\cdot) \in \widetilde{H}^{1}(\Gamma), x_{t}(0, \cdot)=x^{1}(\cdot) \in L_{2}(\Omega)
\end{aligned}
$$


and corresponding to (23) the functional

$$
\begin{aligned}
J^{F}(x)= & \int_{0}^{T} \int_{\Omega}\left(\frac{1}{2}|\nabla x(t, y)|^{2}-\frac{1}{2}\left|x_{t}(t, y)\right|^{2}\right) d y d t \\
& +\int_{0}^{T} \int_{\Omega}\left(F(t, y, x(t, y))-x(t, y) G_{x}(t, y, x(t, y))\right) d y d t \\
& -\left(x^{0}(\cdot), x^{1}(\cdot)\right)_{L_{2}(\Omega)}
\end{aligned}
$$

defined on $U$. Notice that (23) is not the Euler-Lagrange equation for the action functional $J^{F}$.

The dual functional to (24), for any given $w \in U$, now reads

$$
\begin{aligned}
J_{D}^{F}(p, q)= & -\int_{0}^{T} \int_{\Omega} F^{*}\left(t, y,\left(p_{t}(t, y)-\operatorname{div} q(t, y)+G_{x}(t, y, w(t, y))\right)\right) d y d t \\
& -\frac{1}{2} \int_{0}^{T}\|q(t, \cdot)\|_{L_{2}(\Omega)}^{2} d t+\frac{1}{2} \int_{0}^{T}\|p(t, \cdot)\|_{L_{2}(\Omega)}^{2} d t \\
& -(\bar{x}(T, \cdot), p(T, \cdot))_{L_{2}(\Omega)}-\int_{\Sigma} x_{\Gamma}(t, y)\langle q(t, y), v(y)\rangle d y d t
\end{aligned}
$$

for a.e. $t \in[0, T], p_{t}(T-t, \cdot)+\operatorname{div} q(t, \cdot)$ is an element of $L_{2}(\Omega)$ and $F^{*}(t, y, \cdot)$ is Fenchel conjugate to $x \rightarrow F(t, y, x)$, hence

$$
\begin{aligned}
& \int_{0}^{T} \int_{\Omega} F^{*}\left(t, y, p_{t}(t, y)-\operatorname{div} q(t, y)+G_{x}(t, y, w(t, y))\right) d y d t \\
& =\sup _{v \in L_{2}((0, T) \times \Omega)}\left\{\int_{0}^{T} \int_{\Omega}\left(p_{t}(t, y)-\operatorname{div} q(t, y)+G_{x}(t, y, w(t, y))\right) v(t, y) d y d t\right. \\
& \left.\quad-\int_{0}^{T} \int_{\Omega} F(t, y, v(t, y)) d y d t\right\}
\end{aligned}
$$

and

$$
J_{D}^{F}: U_{D}=\left\{\begin{array}{c}
(p, q): p \in C\left([0, T] ; L_{2}(\Omega)\right), \quad p_{t}(\cdot, \cdot)-\operatorname{div} q(\cdot, \cdot) \in \\
L^{1}\left(0, T ; L_{2}(\Omega)\right), \quad p(0, \cdot)=x^{1}(\cdot), \quad q \in L_{2}\left(0, T ;\left(L_{2}(\Omega)\right)^{n}\right)
\end{array}\right\} \rightarrow \mathbf{R} .
$$

We prove the following

Lemma 1 There exist constants $C_{1}^{w}, C_{2}^{w}, C_{3}^{w}, C_{4}^{w}$ independent on $x \in X^{F}$ such that

$$
\begin{aligned}
\|v\|_{L_{2}\left(0, T ; H^{1}(\Omega)\right)} & \leq C_{2}^{w},\left\|v_{t}\right\|_{L_{2}\left(0, T ; L_{2}(\Omega)\right)} \leq C_{1}^{w}, \\
\left\|v_{t t}\right\|_{L_{2}\left(0, T ; H^{-1}(\Omega)\right)} & \leq C_{3}^{w},\|\Delta v\|_{L_{2}\left(0, T ; H^{-1}(\Omega)\right)} \leq C_{4}^{w}, \\
\|v\|_{C\left([0, T] ; H^{1}(\Omega)\right)} & \leq C\left(E_{F}+E_{G}\right)+E^{w}, \\
\left\|v_{t}\right\|_{C\left([0, T] ; L_{2}(\Omega)\right)} & \leq D\left(E_{F}+E_{G}\right)+A^{w} \\
\|v\|_{U} & \leq(C+D)\left(E_{F}+E_{G}\right)+\left(E^{w}+A^{w}\right),
\end{aligned}
$$

where $v$ is the weak solution of the problem

$$
\begin{aligned}
v_{t t}(t, y)-\Delta v(t, y) & =-F_{x}(t, y, x(t, y))+G_{x}(t, y, x(t, y)) \text { in }(0, T) \times \Omega, \\
v(0, y) & =x^{0}(y), \quad y \in \Omega, \\
v_{t}(0, y) & =x^{1}(y), \quad x^{0} \in \widetilde{H}^{1}(\Gamma), \quad x^{1} \in L_{2}(\Omega), \\
v(t, y) & =x_{\Gamma}(t, y), \quad(t, y) \in \Sigma
\end{aligned}
$$


with $x \in X^{F}$.

Proof Fix arbitrary $x \in X^{F}$. Since $x \in U$ and by the assumptions on $F$ and $G$, see (As), it follows that $F_{X}(\cdot, \cdot, x(\cdot, \cdot)), G_{x}(\cdot, \cdot, x(\cdot, \cdot)) \in L^{1}\left(0, T ; L_{2}(\Omega)\right)$. Hence by Theorem 1 and (10) there exists a unique solution $v \in U$ of our problem for the Eq. (26) satisfying $\|v\|_{C\left([0, T] ; H^{1}(\Omega)\right)} \leq C\left(E_{F}+E_{G}\right)+E^{w}$. Taking into account the definition of the set $X^{F}$ we get the following estimation, independent of $x \in X^{F}$

$$
\|v\|_{L_{2}\left(0, T ; H^{1}(\Omega)\right)} \leq T C\left(E_{F}+E_{G}\right)+T E^{w},
$$

by (11)

$$
\left\|v_{t}\right\|_{L_{2}\left(0, T ; L_{2}(\Omega)\right)} \leq T D\left(E_{F}+E_{G}\right)+T A^{w}
$$

and since for some $E_{1}:\|\Delta v\|_{L_{2}\left(0, T ; H^{-1}(\Omega)\right)} \leq E_{1}\|v\|_{L_{2}\left(0, T ; H^{1}(\Omega)\right)}$ thus

$$
\begin{aligned}
\left\|v_{t t}\right\|_{L_{2}\left(0, T ; H^{-1}(\Omega)\right) \leq} & E_{1} T C\left(E_{F}+E_{G}\right) \\
& +E_{1} T E^{w}+\left(E_{F}+E_{G}\right) / T .
\end{aligned}
$$

Hence, putting

$$
\begin{aligned}
& C_{1}^{w}=T D\left(E_{F}+E_{G}\right)+T A^{w}, \\
& C_{2}^{w}=T C\left(E_{F}+E_{G}\right)+T E^{w}, \\
& C_{3}^{w}=E_{1} T C\left(E_{F}+E_{G}\right)+E_{1} T E^{w}+\left(E_{F}+E_{G}\right) / T
\end{aligned}
$$

and

$$
C_{4}^{w}=E_{1} T C\left(E_{F}+E_{G}\right)+E_{1} T E^{w}
$$

we infer the first assertion of the lemma. Further by (10) and (11) we get

$$
\begin{aligned}
\|v\|_{C\left([0, T] ; H^{1}(\Omega)\right)} & \leq C\left(E_{F}+E_{G}\right)+E^{w}, \\
\left\|v_{t}\right\|_{C\left([0, T] ; L_{2}(\Omega)\right)} & \leq D\left(E_{F}+E_{G}\right)+A^{w}
\end{aligned}
$$

and we get the last assertion of the lemma.

Proposition 3 For every $x \in X^{F}$ the weak solution $\tilde{x}$ of the problem

$$
\begin{aligned}
& \tilde{x}_{t t}(t, y)-\Delta \widetilde{x}(t, y)=-F_{x}(t, y, x(t, y))+G_{x}(t, y, x(t, y)), \\
& \tilde{x}(0, y)=x^{0}(y), \tilde{x}_{t}(0, y)=x^{1}(y), y \in \Omega, x^{0} \in \widetilde{H}^{1}(\Gamma), x^{1} \in L_{2}(\Omega), \\
& \tilde{x}(t, y)=x_{\Gamma}(t, y),(t, y) \in \Sigma
\end{aligned}
$$

belongs to $X^{F}$.

Proof Fix arbitrary $x \in X^{F}, F_{x}(\cdot, \cdot, x(\cdot, \cdot)), G_{x}(\cdot, \cdot, x(\cdot, \cdot)) \in L^{1}\left(0, T ; L_{2}(\Omega)\right)$. Hence by Theorem 1 there exists a unique weak solution $\tilde{x} \in U$ of problem (27). Moreover $\tilde{x}_{t t}-\Delta \tilde{x} \in$ $L^{1}\left(0, T ; L_{2}(\Omega)\right)$. By the definition of the set $X^{F}$, it follows that

$$
\begin{gathered}
\left\|F_{x}(\cdot, \cdot, x(\cdot, \cdot))\right\|_{L^{1}\left(0, T ; L_{2}(\Omega)\right)} \leq E_{F}, \\
\left\|G_{x}(\cdot, \cdot, x(\cdot, \cdot))\right\|_{L^{1}\left(0, T ; L_{2}(\Omega)\right)} \leq E_{G} .
\end{gathered}
$$

Further by (10) we get

$$
\begin{aligned}
& \|\widetilde{x}\|_{C\left([0, T] ; H^{1}(\Omega)\right)} \leq C\left(E_{F}+E_{G}\right)+E^{w}, \\
& \left\|\widetilde{x}_{t}\right\|_{C\left([0, T] ; L_{2}(\Omega)\right)} \leq D\left(E_{F}+E_{G}\right)+A^{w}
\end{aligned}
$$


and hence

$$
\|\widetilde{x}\|_{U} \leq(C+D)\left(E_{F}+E_{G}\right)+\left(E^{w}+A^{w}\right) .
$$

Thus $\tilde{x} \in X^{F}$.

Remark 5 Proposition 3 apparently may suggest that it is more convenient to apply a suitable fixed point theorem in order to get the existence of weak solutions to problem (1). Indeed, the above mentioned proposition states that the map $\mathcal{H}$ assigning to $x \in X^{F}$ a weak solution $\tilde{x} \in X^{F}$ of (27) has the property $\mathcal{H}\left(X^{F}\right) \subset X^{F}$. However this is only starting point in fixed point theory. In order to proceed with the so called topological method we must prove that the map $\mathcal{H}$ and set $\mathcal{H}\left(X^{F}\right)$ possess suitable properties. However the assumptions (As) do not imply directly (if at all) neither that $\mathcal{H}$ is contraction nor that $\mathcal{H}\left(X^{F}\right)$ is convex or relatively compact. We have chosen variational approach which ensures not only the existence of solutions but also certain variational properties of solutions which are absolutely new in that case.

Lemma 2 Each $x \in \bar{X}^{F}$ has the weak derivatives $x_{t t}, \Delta x \in L_{2}\left(0, T ; H^{-1}(\Omega)\right)$. Moreover, the sets

$$
X_{t t}=\left\{x_{t t}: x \in \bar{X}^{F}\right\}, \quad X_{\Delta}=\left\{\Delta x: x \in \bar{X}^{F}\right\}
$$

are bounded in $L_{2}\left(0, T ; H^{-1}(\Omega)\right)$. Hence each sequence $\left\{x_{t t}^{j}\right\}$ from $X_{t t}$ has a subsequence converging weakly in $L_{2}\left(0, T ; H^{-1}(\Omega)\right)$ to a certain element from set $X_{t t}$ and sequence $\left\{x_{t}^{j}\right\}$ converges strongly in $L_{2}\left(0, T ; L_{2}(\Omega)\right)$.

Proof It is clear, by Lemma 1 , that $\left\{x_{t t}^{j}\right\}$ from $X_{t t}$ has a subsequence converging weakly in $L_{2}\left(0, T ; H^{-1}(\Omega)\right)$. By the same lemma corresponding sequence $\left\{x^{j}\right\}$ is also weakly (up to some subsequence) converging in $L_{2}\left(0, T ; H^{1}(\Omega)\right)$ to some element $\bar{x}$. By the definition of $X^{F}$ and uniqueness of weak solutions of (27) we infer that $\left\{x_{t t}^{j}\right\}$ has a subsequence weakly convergent to $\bar{x}_{t t}$. The last, the fact that $\left\{x_{t}^{j}\right\}$ is bounded in $L_{2}\left(0, T ; L_{2}(\Omega)\right)$ and a known theorem imply that for this subsequent $\left\{x_{t}^{j}\right\}$ is convergent strongly in $L_{2}\left(0, T ; L_{2}(\Omega)\right)$ to $\bar{x}_{t}$.

We observe that functional $J^{F}$ is well defined on $X^{F}$. Moreover we have

Lemma 3 There exist constants $M_{1}^{F}, M_{2}^{F}$ such that

$$
\begin{aligned}
& M_{1}^{F} \leq \int_{0}^{T} \int_{\Omega} F(t, y, x(t, y)) d y d t \leq M_{2}^{F}, \\
& M_{1}^{F} \leq \int_{0}^{T} \int_{\Omega} G(t, y, x(t, y)) d y d t \leq M_{2}^{F}
\end{aligned}
$$

for all $x \in X^{F}$.

Proof Note that by the construction of $X^{F}$ it is a ball in $U$, we have also that $U \subset H^{1}((0, T) \times$ $\Omega)$. It is easy to see that $X^{F}$ is weakly compact in $H^{1}((0, T) \times \Omega)$ and that the functionals $x \rightarrow \int_{0}^{T} \int_{\Omega} F(t, y, x(t, y)) d y d t, x \rightarrow \int_{0}^{T} \int_{\Omega} G(t, y, x(t, y)) d y d t$ are weakly continuous in $H^{1}((0, T) \times \Omega)$ (weakly converging sequence in $H^{1}((0, T) \times \Omega)$ is strongly convergent in $\left.L^{2}((0, T) \times \Omega)\right)$. Hence we infer the assertions of the Lemma. 
Lemma 4 The functional $J^{F}$ attains its maximum in $X^{F}$ i.e.

$$
\sup _{x \in X^{F}} J^{F}(x)=J^{F}(\bar{x})
$$

where $\bar{x} \in X^{F}$.

Proof By definition of the set $X^{F}$ and Lemma 3 we see that the functional $J^{F}$ is bounded in $X^{F}$. We denote by $\left\{x^{j}\right\}$ a maximizing sequence for $J^{F}$ in $X^{F}$. This sequence has a subsequence which we denote again by $\left\{x^{j}\right\}$ converging weakly in $L_{2}\left(0, T ; H^{1}(\Omega)\right)$ and strongly in $L_{2}\left(0, T ; L_{2}(\Omega)\right)$, hence also strongly in $L_{2}((0, T) \times \Omega ; \mathbf{R})$ to a certain element $\bar{x} \in U$. Moreover $\left\{x^{j}\right\}$ is also convergent almost everywhere. Thus by the construction of the set $X^{F}$ and uniqueness of weak solutions of (27) we observe that $\bar{x} \in X^{F}$. Hence

$$
\limsup _{j \rightarrow \infty} J^{F}\left(x^{j}\right) \leq J^{F}(\bar{x}) .
$$

Thus

$$
\sup _{x^{j} \in X^{F}} J^{F}\left(x^{j}\right)=J^{F}(\bar{x}) .
$$

To consider properly the dual action functional let us put

$$
\begin{aligned}
W_{t}^{1 F}= & \left\{p \in C\left([0, T] ; L_{2}(\Omega)\right):\right. \\
& \left.p_{t} \in L^{2}\left(0, T ; H^{-1}(\Omega)\right), p(0, \cdot)=x^{1}(\cdot)\right\}
\end{aligned}
$$

and

$$
\begin{aligned}
& W_{y}^{1 F}=\left\{q \in L_{2}\left(0, T ;\left(L_{2}(\Omega)\right)^{n}\right): \text { there exists } p \in W_{t}^{1 F}\right. \\
&\text { such that } \left.p_{t}(\cdot, \cdot)-\operatorname{div} q(\cdot, \cdot) \in L_{2}\left(0, T ; L_{2}(\Omega)\right)\right\} .
\end{aligned}
$$

and define a set on which a dual functional will be considered.

Definition of $X^{F d}$ We say that an element $(p, q) \in W_{t}^{1 F} \times W_{y}^{1 F}$ belongs to $X^{F d}$ provided that there exists $x \in X^{F}$ such that for a.e. $t \in(0, T)$

$$
p_{t}(t, \cdot)-\operatorname{div} q(t, \cdot)=-F_{x}(t, \cdot, x(t, \cdot))+G_{x}(t, \cdot, x(t, \cdot))
$$

with

$$
q(t, \cdot)=\nabla x(t, \cdot) .
$$

We observe that neither $X^{F}$ nor $X^{F d}$ is a linear space. Thus even standard calculations using convexity arguments are rather difficult. What helps us is a special structure of the sets $X^{F}$ and $X^{F d}$ which despite their nonlinearity makes these calculations possible.

Now we may state the main result of the paper which is the following existence theorem.

Theorem 5 There is $\bar{x} \in X^{F}$, such that $\sup _{x \in X^{F}} J^{F}(x)=J^{F}(\bar{x})$. Moreover, there is $(\bar{p}, \bar{q}) \in X^{F d}$ such that

$$
J_{D}^{F}(\bar{p}, \bar{q})=\sup _{x \in X^{F}} J^{F}(x)=J^{F}(\bar{x})
$$


and the following system holds, for $t \in[0, T]$,

$$
\begin{aligned}
& \bar{x}_{t}(t, \cdot)=\bar{p}(t, \cdot), \\
& \nabla \bar{x}(t, \cdot)=\bar{q}(t, \cdot), \\
& \bar{p}_{t}(t, \cdot)-\operatorname{div} \bar{q}(t, \cdot)=-F_{x}(t, \cdot, \bar{x}(t, \cdot))+G_{x}(t, \cdot, \bar{x}(t, \cdot)) .
\end{aligned}
$$

\subsection{Variational Principle: The Case $l=F_{x}-G_{x}$}

We state the necessary conditions. We observe that due to the construction of the set $X^{F}$ and Lemma 2 it follows that a maximizing sequence in $X^{F}$ for the functional $J^{F}$ may be assumed to be weakly convergent in $L_{2}\left(0, T ; H^{1}(\Omega)\right)$ and strongly in $L_{2}\left(0, T ; L_{2}(\Omega)\right)$.

Theorem 6 Let $\sup _{x \in X^{F}} J^{F}(x)=J^{F}(\bar{x})$, where $\bar{x} \in L_{2}\left(0, T ; H^{1}(\Omega)\right)$ is a limit, strong in $L_{2}\left(0, T ; L_{2}(\Omega)\right)$ and weak in $L_{2}\left(0, T ; H^{1}(\Omega)\right)$, of a maximizing sequence $\left\{x^{j}\right\} \subset X^{F}$. Then there exists $(\bar{p}, \bar{q}) \in X^{F d}$ such that for a.e. $t \in(0, T)$,

$$
\begin{aligned}
& \bar{p}(t, \cdot)=\bar{x}_{t}(t, \cdot), \\
& \bar{q}(t, \cdot)=\nabla \bar{x}(t, \cdot), \\
& \bar{p}_{t}(t, \cdot)-\operatorname{div} \bar{q}(t, \cdot)+F_{x}(t, \cdot, \bar{x}(t, \cdot))-G_{x}(t, \cdot, \bar{x}(t, \cdot))=0
\end{aligned}
$$

and

$$
J^{F}(\bar{x})=J_{D}^{F}(\bar{p}, \bar{q}) .
$$

Proof Let $\bar{x} \in X^{F}$ be such that $J^{F}(\bar{x})=\sup _{x^{j} \in X^{F}} J^{F}\left(x^{j}\right)$. Define

$$
\bar{p}_{t}(t, \cdot)=\operatorname{div} \bar{q}(t, \cdot)-F_{x}(t, \cdot, \bar{x}(t, \cdot))+G_{x}(t, \cdot, \bar{x}(t, \cdot)), \quad t \in(0, T)
$$

with $\bar{q}$ given by

$$
\bar{q}(t, y)=\nabla \bar{x}(t, y) \text { for a.e. }(t, y) \in(0, T) \times \Omega .
$$

By the definitions of $J^{F}, J_{D}^{F}$, relations (37), (36) and the Fenchel-Young inequality it follows that

$$
\begin{aligned}
J^{F}(\bar{x})= & \int_{0}^{T} \int_{\Omega}\left(\frac{1}{2}|\nabla \bar{x}(t, y)|^{2}-\frac{1}{2}\left|\bar{x}_{t}(t, y)\right|^{2}+F(t, y, \bar{x}(t, y))\right) d y d t \\
& -\int_{0}^{T} \int_{\Omega} \bar{x}(t, y) G_{x}(t, y, \bar{x}(t, y)) d y d t-\left(x^{0}(\cdot), x^{1}(\cdot)\right)_{L_{2}(\Omega)} \\
\leq & -\int_{0}^{T} \int_{\Omega} \bar{x}_{t}(t, y) \bar{p}(t, y) d y d t+\int_{0}^{T} \int_{\Omega}\langle\nabla \bar{x}(t, y), \bar{q}(t, y)\rangle d y d t \\
& +\frac{1}{2} \int_{0}^{T} \int_{\Omega}|\bar{p}(t, y)|^{2} d y d t-\frac{1}{2} \int_{0}^{T} \int_{\Omega}|\bar{q}(t, y)|^{2} d y d t \\
& +\int_{0}^{T} \int_{\Omega}\left(F(t, y, \bar{x}(t, y))-\bar{x}(t, y) G_{x}(t, y, \bar{x}(t, y))\right) d y d t \\
= & -\int_{0}^{T} \int_{\Omega} F^{*}\left(t, y,\left(-\bar{p}_{t}(t, y)+d i v \bar{q}(t, y)+G_{x}(t, y, \bar{x}(t, y))\right)\right) d y d t \\
& -\frac{1}{2} \int_{0}^{T} \int_{\Omega}|\bar{q}(t, y)|^{2} d y d t-(\bar{x}(T, \cdot), \bar{p}(T, \cdot))_{L_{2}(\Omega)} \\
& +\frac{1}{2} \int_{0}^{T} \int_{\Omega}|\bar{p}(t, y)|^{2} d y d t-\int_{\Sigma} x_{\Gamma}(t, y)\langle\bar{q}(t, y), v(y)\rangle d y d t .
\end{aligned}
$$


Therefore we get that

$$
J^{F}(\bar{x}) \leq J_{D}^{F}(\bar{p}, \bar{q})
$$

Let $\hat{x} \in X^{F}$ denote the element corresponding to $\bar{p}$ accordingly to the definition of the set $X^{F d}$ i.e. $\hat{x}_{t}=\bar{p}$. It is clear that then $J^{F}(\hat{x}) \leq J^{F}(\bar{x})$. We observe that by the Fenchel-Young equality we have

$$
\begin{aligned}
J^{F}(\bar{x})= & \sup _{x \in X^{F}} J^{F}(x) \geq J^{F}(\hat{x}) \\
= & \int_{0}^{T} \int_{\Omega}\left(\frac{1}{2}|\nabla \hat{x}(t, y)|^{2}-\frac{1}{2}\left|\hat{x}_{t}(t, y)\right|^{2}\right) d y d t-\left(x^{0}(\cdot), x^{1}(\cdot)\right)_{L_{2}(\Omega)} \\
& +\int_{0}^{T} \int_{\Omega}\left(F(t, y, \hat{x}(t, y))-\hat{x}(t, y) G_{x}(t, y, \hat{x}(t, y))\right) d y d t \\
\geq & -\frac{1}{2} \int_{0}^{T} \int_{\Omega}|\bar{q}(t, y)|^{2} d y d t+\int_{0}^{T} \int_{\Omega}\langle\nabla \hat{x}(t, y), \bar{q}(t, y)\rangle d y d t \\
& +\frac{1}{2} \int_{0}^{T} \int_{\Omega}|\bar{p}(t, y)|^{2} d y d t+\int_{0}^{T}\left(\hat{x}(t, \cdot), \bar{p}_{t}(t, \cdot)\right)_{H^{1}, H^{-1}} d t \\
& +\int_{0}^{T} \int_{\Omega}\left(F(t, y, \hat{x}(t, y))-\hat{x}(t, y) G_{x}(t, y, \hat{x}(t, y))\right) d y d t \\
& -(\hat{x}(T, \cdot), \hat{p}(T, \cdot))_{L_{2}(\Omega)} \\
\geq & -\frac{1}{2} \int_{0}^{T} \int_{\Omega}|\bar{q}(t, y)|^{2} d y d t+\frac{1}{2} \int_{0}^{T} \int_{\Omega}|\bar{p}(t, y)|^{2} d y d t \\
& +\inf _{x \in X^{F}}\left\{\int_{0}^{T} \int_{\Omega} F(t, y, x(t, y)) d y d t-(x(T, \cdot), p(T, \cdot))_{L_{2}(\Omega)}\right. \\
& \left.-\int_{0}^{T} \int_{\Omega} x(t, y)\left(d i v \bar{q}(t, y)-\bar{p}_{t}(t, y)+G_{x}(t, y, x(t, y))\right) d y d t\right\} \\
& -\int_{\Sigma} x_{\Gamma}(t, y)\langle\bar{q}(t, y), v(y)\rangle d y d t=J_{D}^{F}(\bar{p}, \bar{q}) . \\
& \\
&
\end{aligned}
$$

Inequality $J^{F}(\bar{x}) \geq J_{D}^{F}(\bar{p}, \bar{q})$ and (38) imply equality $J^{F}(\bar{x})=J_{D}^{F}(\bar{p}, \bar{q})$. Moreover $J^{F}(\bar{x})=J_{D}^{F}(\bar{p}, \bar{q})$, implies

$$
\begin{aligned}
\int_{0}^{T} & \int_{\Omega} F^{*}\left(t, y,\left(-\bar{p}_{t}(t, y)+\operatorname{div} \bar{q}(t, y)+G_{x}(t, y, \bar{x}(t, y))\right)\right) d y d t \\
& +\int_{0}^{T} \int_{\Omega}\left(F(t, y, \bar{x}(t, y))-\bar{x}(t, y) G_{x}(t, y, \bar{x}(t, y))\right) d y d t \\
& +\frac{1}{2} \int_{0}^{T} \int_{\Omega}|\bar{q}(t, y)|^{2} d y d t+\frac{1}{2} \int_{0}^{T} \int_{\Omega}|\nabla \bar{x}(t, y)|^{2} d y d t \\
= & \frac{1}{2} \int_{0}^{T} \int_{\Omega}\left|\bar{x}_{t}(t, y)\right|^{2} d y d t+\frac{1}{2} \int_{0}^{T} \int_{\Omega}|\bar{p}(t, y)|^{2} d y d t \\
& -\int_{\Sigma} x_{\Gamma}(t, y)(\bar{q}(t, y), v(y)) d y d t+\left(x^{0}(\cdot), x^{1}(\cdot)\right)_{L^{2}(\Omega)}-(\bar{x}(T, \cdot), \bar{p}(T, \cdot))_{L_{2}(\Omega)} .
\end{aligned}
$$


Therefore by (37), (36) and standard convexity arguments

$$
\begin{aligned}
& \frac{1}{2} \int_{0}^{T} \int_{\Omega}|\bar{p}(t, y)|^{2} d y d t+\frac{1}{2} \int_{0}^{T} \int_{\Omega}\left|\bar{x}_{t}(t, y)\right|^{2} d y d t \\
& \quad=\int_{0}^{T} \int_{\Omega} \bar{x}_{t}(t, y) \bar{p}(t, y) d y d t .
\end{aligned}
$$

Hence by the Fenchel-Young transform we obtain that $\bar{p}(t, y)=\bar{x}_{t}(t, y)$ i.e. (33).

\subsection{Variational Principle: The Case $l=-F_{x}-G_{x}$}

A similar theorem is true for the problem

$$
\begin{aligned}
& x_{t t}(t, y)-\Delta x(t, y)-F_{x}(t, y, x(t, y))-G_{x}(t, y, x(t, y))=0, \text { in }(0, T) \times \Omega, \\
& x(t, y)=x_{\Gamma}(t, y), \quad(t, y) \in \Sigma, \\
& x(0, \cdot)=x^{0}(\cdot) \in \widetilde{H}^{1}(\Gamma), x_{t}(0, \cdot)=x^{1}(\cdot) \in L_{2}(\Omega)
\end{aligned}
$$

and corresponding to it functional

$$
\begin{aligned}
J^{F-}(x)= & \int_{0}^{T} \int_{\Omega}\left(\frac{1}{2}|\nabla x(t, y)|^{2}-\frac{1}{2}\left|x_{t}(t, y)\right|^{2}-F(t, y, x(t, y))\right) d y d t \\
& -\int_{0}^{T} \int_{\Omega} x(t, y) G_{x}(t, y, x(t, y)) d y d t-\left(x^{0}(\cdot), x^{1}(\cdot)\right)_{L_{2}(\Omega)},
\end{aligned}
$$

defined on $U$ with same hypotheses (As) and the set $X^{F}$. Really, Lemmas 1-4 are still valid as sign of $F$ does not change their proofs, also the proof of the above theorem does not change. Hence we get for (39) the following theorem.

Theorem 7 There are $\bar{x} \in X^{F}$ and $(\bar{p}, \bar{q}) \in L_{2}\left(0, T ; L_{2}(\Omega)\right) \times L_{2}\left(0, T ; L_{2}(\Omega)\right)$ such that for a.e. $(t, y) \in(0, T) \times \Omega$,

$$
\begin{aligned}
& \bar{p}(t, y)=\bar{x}_{t}(t, y), \\
& \bar{q}(t, y)=\nabla \bar{x}(t, y), \\
& \bar{p}_{t}(t, \cdot)-\operatorname{div} \bar{q}(t, \cdot)-F_{x}(t, \cdot, \bar{x}(t, \cdot))-G_{x}(t, \cdot, \bar{x}(t, \cdot))=0
\end{aligned}
$$

and

$$
J^{F-}(\bar{x})=J_{D}^{F-}(\bar{p}, \bar{q}),
$$

where

$$
\begin{aligned}
J_{D}^{F-}(\bar{p}, \bar{q})= & \int_{0}^{T} \int_{\Omega} F^{*}\left(t, y,\left(-\bar{p}_{t}(t, y)+\operatorname{div} \bar{q}(t, y)+G_{x}(t, y, \bar{x}(t, y))\right)\right) d y d t \\
& -\frac{1}{2} \int_{0}^{T} \int_{\Omega}|\bar{q}(t, y)|^{2} d y d t+\frac{1}{2} \int_{0}^{T} \int_{\Omega}|\bar{p}(t, y)|^{2} d y d t \\
& -\int_{\Sigma} x_{\Gamma}(t, y)\langle\bar{q}(t, y), v(y)\rangle d y d t-(\bar{x}(T, \cdot), \bar{p}(T, \cdot))_{L_{2}(\Omega)} .
\end{aligned}
$$

\subsection{Proof of Stability: Theorem 3}

In this section we prove some stability results in our nonlinear case i.e. Theorem 3 . To this effect let us assume we are given a sequence $\left\{x_{n}^{0}\right\}$ in $\widetilde{H}^{1}(\Gamma)$ converging to $\bar{x}^{0}$ in $\widetilde{H}^{1}(\Gamma)$. 
Let $\left\{x_{\Gamma n}\right\}$ be a sequence of solutions to (5) corresponding to $\left\{x_{n}^{0}\right\}$. Then by Proposition 2 and its proof we know that $J_{\Gamma}^{t}\left(x_{\Gamma n}\right)=0, t \in[0, T], n=1,2, \ldots$ and that $J_{\Gamma}^{t}(x) \geq 0$ for all $x \in U^{t}, t \in[0, T]$ (moreover $J_{\Gamma}^{t}$ is weakly lower semicontinuous in $U^{t}, t \in[0, T]$ ). Therefore the sequence $\left\{x_{\Gamma n}\right\}$ is bounded in $\widetilde{H}^{1}(\Gamma)$ and hence it has a subsequence (which we shall denote again by $\left.\left\{x_{\Gamma n}\right\}\right)$ converging weakly in $\widetilde{H}^{1}(\Gamma)$ to some $x_{\Gamma} \in \widetilde{H}^{1}(\Gamma)$ and $J_{\Gamma}^{t}\left(x_{\Gamma}\right)=0$ on $U^{t}, t \in[0, T]$. The last implies that $x_{\Gamma}$ is a solution to (5) corresponding to $\bar{x}^{0}$.

Next let us assume that $\left\{x_{n}^{0}\right\},\left\{x_{n}^{1}\right\}$ are given sequences in $\widetilde{H}^{1}(\Gamma), L_{2}(\Omega)$, respectively and such that

$$
\left\|x_{n}^{0}\right\|_{C\left([0, T] ; H^{1}(\Omega)\right)} \leq C\left(E_{F}+E_{G}\right)+E^{w}, \quad\left\|x_{n}^{1}\right\|_{C\left([0, T] ; L_{2}(\Omega)\right)} \leq C\left(E_{F}+E_{G}\right)+E^{w},
$$

$n=1,2 \ldots$, converging to $\bar{x}^{0}, \bar{x}^{1}$ in $H^{1}(\Omega), L_{2}(\Omega)$ and $x_{n}^{0}(y)=x_{\Gamma n}(0, y)$ on $\Gamma$, $n=1,2, \ldots$ Thus we assume hypotheses $(\mathbf{H} \Gamma),(\mathbf{A s})$ to be considered and satisfied. In consequence, all the assertions of Theorem 4 are true for all $n$ with estimations independent on $n$. Because, we have nonlinear problem we cannot expect the same type of continuous dependence as in [13] for linear case. First note that for each $x_{n}^{0}, x_{n}^{1}$ there exists a solution $\bar{x}_{n} \in X^{F} \subset U$ to (1) determined by $x_{n}^{0}$ and $x_{n}^{1}, n=1,2, \ldots$ Therefore, choosing suitable subsequence, let $\bar{x} \in X^{F}$ be a weak limit in $H^{1}((0, T) \times \Omega)$ and strong in $L_{2}((0, T) \times \Omega)$ of $\left\{\bar{x}_{n}\right\}$. We know also that for all $n, J^{F}\left(\bar{x}_{n}\right)=J_{D}^{F}\left(\bar{p}_{n}, \bar{q}_{n}\right)$ where $\left\{\bar{p}_{n}, \bar{q}_{n}\right\}$ denote the pair of sequences corresponding to $\left\{\bar{x}_{n}\right\}$ and satisfying

$$
\begin{aligned}
& \bar{x}_{n t}(t, \cdot)=\bar{p}_{n}(t, \cdot), \\
& \nabla \bar{x}_{n}(t, \cdot)=\bar{q}_{n}(t, \cdot), \\
& -\bar{p}_{n t}(t, \cdot)+\operatorname{div} \bar{q}_{n}(t, \cdot)-l\left(t, y, \bar{x}_{n}(t, \cdot)\right)=0 .
\end{aligned}
$$

As $\left\{\bar{x}_{n}\right\}$ is weakly convergent in $H^{1}((0, T) \times \Omega)$ and strongly in $L_{2}\left(0, T ; L_{2}(\Omega)\right), \bar{x}_{n t} \rightarrow$ $\bar{x}_{t}=\bar{p}, \nabla \bar{x}_{n} \rightarrow \nabla \bar{x}=\bar{q}$ in $L_{2}\left(0, T ; L_{2}(\Omega)\right)$, therefore $\bar{x}_{n}$ converges pointwise to $\bar{x}$. Hence $\left\{-\bar{p}_{n t}+\operatorname{div} \bar{q}_{n}\right\}$ converges pointwise to $-\bar{p}_{t}+\operatorname{div} \bar{q}$. Thus we infer that

$$
J^{F}(\bar{x})=\lim _{n \rightarrow \infty} J^{F}\left(x_{n}\right)=\lim _{n \rightarrow \infty} J_{D}^{F}\left(p_{n}, q_{n}\right)=J_{D}^{F}(\bar{p}, \bar{q})
$$

and in consequence

$$
\begin{aligned}
& \bar{x}_{t}(t, \cdot)=\bar{p}(t, \cdot), \\
& \nabla \bar{x}(t, \cdot)=\bar{q}(t, \cdot), \\
& -\bar{p}_{t}(t, \cdot)+\operatorname{div} \bar{q}(t, \cdot)-l(t, y, \bar{x}(t, \cdot))=0
\end{aligned}
$$

and so we get the assertion of the theorem.

Open Access This article is distributed under the terms of the Creative Commons Attribution 4.0 International License (http://creativecommons.org/licenses/by/4.0/), which permits unrestricted use, distribution, and reproduction in any medium, provided you give appropriate credit to the original author(s) and the source, provide a link to the Creative Commons license, and indicate if changes were made.

\section{References}

1. Adams, R.A.: Sobolev Spaces. Academic Press, London (1975)

2. Alves, C.O., Cavalcanti, M.M.: On existence, uniform decay rates and blow up for solutions of the 2-D wave equation with exponential source. Calc. Var. Partial Differ. Equ. 34, 377-411 (2009) 
3. Barbu, V., Lasiecka, I., Rammaha, M.A.: On nonlinear wave equations with degenerate damping and source terms. Trans. Am. Math. Soc. 357, 2571-2611 (2005)

4. Bociu, L., Lasiecka, I.: Uniqueness of weak solutions for the semilinear wave equations with supercritical boundary/interior sources and damping. DCDS 22, 835-860 (2008)

5. Bociu, L., Lasiecka, I.: Local Hadamard well-posedness for nonlinear wave equations with supercritical sources and damping. J. Differ. Equ. (2010). doi:10.1016/j.jde.2010.03.009

6. Bociu, L., Rammaha, M., Toundykov, D.: On a wave equation with supercritical interior and boundary sources and damping terms. Math. Nachr. 284, 2032-2064 (2011)

7. Brezis, H., Ekeland, I.: Un principe variationnel associé à certaines équations paraboliques. Le cas dépendant du temps. C. R. Acad. Sci. Paris Sér. A-B 282(20), Ai, A1197-A1198 (1976) (French)

8. Cavalcanti, M.M., Cavalcanti, V.N.D., Filho, J.S.P., Soriano, J.A.: Existence and uniform decay of solutions of a parabolic-hyperbolic equation with nonlinear boundary damping and boundary source term. Commun. Anal. Geom. 10, 451-466 (2002)

9. Cavalcanti, M.M., Cavalcanti, V.N.D., Lasiecka, I.: Well-posedness and optimal decay rates for the wave equation with nonlinear boundary damping-source interaction. J. Differ. Equ. 236, 407-459 (2007)

10. Cavalcanti, M.M., Cavalcanti, V.N.D., Martinez, P.: Existence and decay rate estimates for the wave equation with nonlinear boundary damping and source term. J. Differ. Equ. 203, 119-158 (2004)

11. Ekeland, I., Temam, R.: Convex Analysis and Variational Problems. Studies in Applied Mathematics, vol. 1. North-Holland, Amsterdam (1976)

12. Glassey, R.T.: Blow-up theorems for nonlinear wave equations. Math. Z. 132, 183-203 (1973)

13. Lasiecka, I., Lions, J.L., Triggiani, R.: Nonhomogeneous boundary value problems for second order hyperbolic operators. J. Math. Pures Appl. 65, 149-192 (1986)

14. Levine, H.A.: Instability and nonexistence of global solutions of nonlinear wave equations of the form $P u_{t t}=A u+F(u)$. Trans. Am. Math. Soc. 192, 1-21 (1974)

15. Mordukhovich, B.S., Raymond, J.P.: Dirichlet boundary control of hyperbolic equations in the presence of state constraints. Appl. Math. Optim. 49, 145-157 (2004)

16. Mordukhovich, B.S., Zhang, K.: Minimax control of parabolic systems with Dirichlet boundary conditions and state constraints. Appl. Math. Optim. 36, 323-360 (1997)

17. Mordukhovich, B.S., Zhang, K.: Dirichlet boundary control of parabolic systems with pointwise state constraints. In: Control and Estimation of Distributed Parameter Systems (Vorau, 1996). International Series of Numerical Mathematics, vol. 126, pp. 223-236. Birkhaeuser, Basel (1998)

18. Nowakowski, A.: Nonlinear parabolic equations associated with subdifferential operators, periodic problems. Bull. Polish Acad. Sci. Math. 16, 615-621 (1998)

19. Nowakowski, A.: Nonhomogeneous boundary value problem for semilinear hyperbolic equation. J. Dyn. Control Syst. 14(4), 537-558 (2008)

20. Nowakowski, A.: Solvability and stability of a semilinear wave equation with nonlinear boundary conditions. Nonlinear Anal. 73(6), 1495-1514 (2010)

21. Nowakowski, A.: Variational approach to stability of semilinear wave equation with nonlinear boundary conditions. DCDS 19, 2603-2616 (2014)

22. Nowakowska, I., Nowakowski, A.: Dirichlet problem for semilinear hyperbolic equation. Multidimensional case. J. Math. Anal. Appl. 338(2), 771-783 (2008)

23. Payne, L.E., Sattinger, D.: Saddle points and instability of nonlinear hyperbolic equations. Israel Math. J. 22, 273-303 (1981)

24. Rammaha, M.A.: The influence of damping and source terms on solutions of nonlinear wave equations. Bol. Soc. Parana. Math. 25, 77-90 (2007)

25. Tsutsumi, H.: On solutions of semilinear differential equations in a Hilbert space. Math. Jpn. 17, 173-193 (1972)

26. Vitillaro, E.: Global existence of the wave equation with nonlinear boundary damping and source terms. J. Differ. Equ. 186, 259-298 (2002)

27. Vitillaro, E.: A potential well theory for the wave equation with nonlinear source and boundary damping terms. Glasg. Math. J. 44, 375-395 (2002)

28. Yordanov, B., Zhang, Q.S.: Finite-time blowup for wave equations with a potential. SIAM J. Math. Anal. 36, 1426-1433 (2005) 